\title{
Advanced Assembly Solutions for the Airbus RACER Joined-Wing Configuration
}

\author{
Author, co-author (Do NOT enter this information. It will be pulled from participant tab in \\ MyTechZone) \\ Affiliation (Do NOT enter this information. It will be pulled from participant tab in MyTechZone)
}

\begin{abstract}
The Rapid And Cost Effective Rotorcraft (RACER) is being developed by Airbus Helicopters (AH) to demonstrate a new Vertical Take-Off and Landing configuration to fill the mobility gap between conventional helicopters and aeroplanes. RACER is a compound rotorcraft featuring wings and multiple rotors. The wing arrangement suggested by AH is defined as a staggered bi-plane joined configuration with an upper and a lower straight wing, either side of the fuselage, connected at their outboard extent to form a triangular structure. The ASTRAL consortium, consisting of the University of Nottingham and GE Aviation Systems, are responsible for the design, manufacture, assembly and testing of the wings. Producing an optimised strategy to assemble a joined-wing configuration for a passenger carrying rotorcraft is challenging and novel. The objective of this work concerns all aspects of assembling the joined-wing structure.
\end{abstract}

The joined-wing and fuselage structures will be produced independently and mated together during the final RACER assembly. A multi-stage process will deliver the joined-wing assembly and ensure it will fit to the fuselage. Producing the individual wing structures requires a novel build philosophy driven by the innovative, one-piece composite moulding that forms the leading edge (LE) and upper aerodynamic surface of each wing. Using the Flap subassembly as the tool to set interfacing wing-box items provides a cost effective solution to assembly jig design. The independent wing structures must be joined at the outboard hinge line, whilst meeting the fuselage interchangeability definition. Matched tooling, replicating the fuselage interface, will be employed to overcome this hurdle. Successfully joining the wing structures also requires the innovative application of $3 \mathrm{D}$ tolerance analysis methods to size bush diameters within the interface.

The overall build philosophy is summarised in this work, including assembly sequencing; definition of datum frames; assembly tooling; design tolerances and the analysis required to deliver assembly key characteristics.

\section{Introduction}

Compound helicopters, featuring lateral rotors as well as a primary rotor, are increasingly seen as the future of rotorcraft design. Capable of supporting a range of service applications, compound rotorcraft have the potential to deliver increased efficiencies and higher speeds relative to traditional rotorcraft. The Rapid And Cost Effective Rotorcraft (RACER) demonstrator [1] is being developed by AH to

Page 1 of 10 further validate the compound rotorcraft configuration. AH have chosen to employ a joined-wing design [2], which increases the stiffness relative to a traditional wing design. The RACER design also includes two lateral rotors, mounted aft of the Nacelles at the outboard extent of the wings, as depicted in Figure 1.

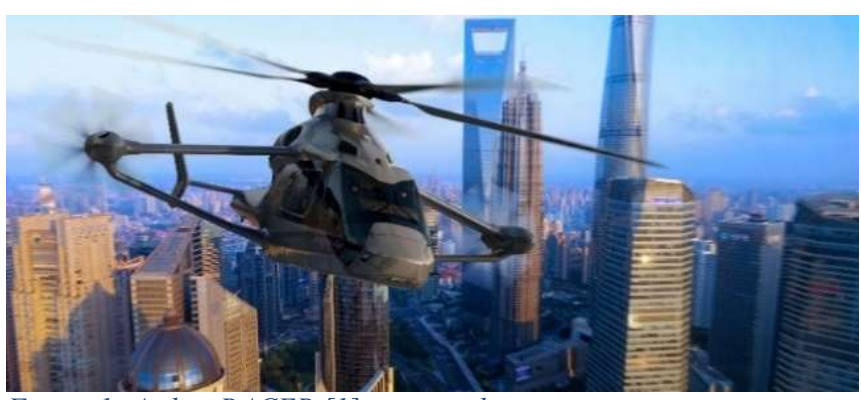

Figure 1: Airbus RACER [1] concept design

The ASTRAL consortium will be delivering one set of wings for flight and a second set for dynamic testing. However, the wings and necessary manufacturing or assembly processes are designed to enable a production rate of 100 aircraft per year. Cost related to the assembly of aerostructures accounts for a significant proportion of the overall cost required to get an aircraft airborne and it can be predicted as a function of the number of parts and fasteners, referred to as 'aircraft general supply' parts (AGS), within a structure [3]. Given the relatively small scale of the RACER wing and the optimised design, it could be assumed that the assembly cost would be low. However, the joined-wing configuration presents unique challenges during the assembly of the wing structures and the connection to the fuselage.

Two assembly methodologies were considered for the RACER wing structures; determinate assembly or traditional assembly set by jigs. Determinate assembly, also known as 'part-to-part' or 'jig-less' assembly relies on the precision manufacturing of a few critical features, within the definition of each child part, that allow interfacing items to be accurately positioned. This approach significantly reduces the importance of an assembly jig, which may only be required to support the mass of the wing structure [4]. Resultant jig structures may be more cost effective given requirements on their ability to set part positions are much less stringent. This approach can also reduce the need for assembly processing, deliver a shorter build duration and reduce the lead-time for assembly tooling [5]. Conversely, the requirement to include precision location features within component definitions can transfer cost into the manufacturing processes. Equally, significant analysis of 
3D tolerance stack-ups must be completed to confirm the assembly can meet geometrical requirements.

The traditional method of assembling wings is to employ jig structures that have been precisely calibrated to ensure they can accurately locate child parts within the wing assembly and allow assembly processing tasks to be completed [6]. Datum features of items within wing assemblies will contact 'pick-ups', within the jig structure, to minimise the impact of manufacturing tolerances on the position of parts within assemblies. This approach reduces the requirement for precision manufactured parts but may transfer cost into the assembly process. The traditional method of assembling wings, using precision jigs, was selected for the production of the RACER wings in order to remove complexity and cost from the manufacture of the novel, composite items within the structures. The design of the tooling required to facilitate assembly of the joinedwing structures is also highlighted.

Employing matched tooling has been proposed to aid the mounting of the joined-wing structure to the fuselage, ensuring the independently built wing structures can be successfully assembled by replicating the interface with the fuselage. The matched tooling configuration was debated and could be designed either as a one-piece tool or a modular version. The decision significantly affects the overall wing assembly processes. Given the complexity of the wing product, threedimensional (3D) tolerance analysis methods were needed to perform the analysis and make an informed decision. Several 3D tolerance methods have been suggested in the literature, e.g. in [7-11]. A comparison of the various $3 \mathrm{D}$ tolerance analysis techniques can be found in [12]. Homogeneous Transformation Method (HTM) [4] was selected to perform the variation propagation analysis. A detailed discussion about the matched tooling concepts as well as the implementation of the HTM technique to RACER wing configuration can be found in [13]

As an overview, this paper describes the optimised philosophy required to produce the RACER joined-wing assembly. An overview of joined-wing configurations and their typical characteristics are noted to provide clarity of the wing architecture. The child structures within the RACER joined-wing assembly are then detailed, including the wing datum systems and the interchangeability requirements, to aid the identification of the assembly challenges. The processes and tooling required to deliver the joined-wing assembly are then outlined in relation to the three key stages within the overall process. Firstly, methods of assembling the independent wing structures are identified and the chosen method is selected. A cost effective solution to produce Flap sub-assemblies and use them as tooling to set components positions is then defined. Finally, the process of assembling the independent wing structures into a joined-wing product is described. This includes details of the analytical assembly models produced to define the necessary size of interfacing bush geometry. The result of this work is the presentation of the RACER joined-wing assembly build philosophy and the identification of tooling required. The completed work is summarised through simplified assembly sequence diagrams.

\section{Overview of RACER Compound Helicopter}

The compound helicopter RACER was developed, as part of the Clean Sky 2 European research programme, in an attempt to fill the mobility gap between conventional helicopters and aeroplanes [1]. This Vertical Take-Off and Landing (VTOL) aircraft should ensure more efficient emergency and search and rescue services as well as improving citizen mobility by offering faster gate to gate passenger Page 2 of 10 transport. Conventional helicopters offer vertical take-off and landing capability but their flight speed, limited by the aerodynamics of the main rotor, is substantially lower than traditionally fixed-winged aircraft. In fact, the stall of the retreating blade and the shock wave generation on the advancing blade bound the rotor rotational speed in cruise, and consequently limit the helicopter maximum advancing speed. The most promising concepts to overcome these limitations are the convertiplane and the compound helicopter [1]. In a convertiplane or tiltrotor, the rotor is tilted perpendicularly to the flight trajectory to provide thrust during cruise flight while a fixedwing provides the required lift. In a compound helicopter, the main rotor is fixed, providing lift and thrust in cruise flight, but fixed wing (lift compounding) and/or thruster (thrust compounding) are added to off-load the rotor during horizontal flight. The reduction of lift and thrust load required from the rotor allows a decrease of its rotational speed and a consequent increment of the aircraft speed limit.

The RACER is a medium class rotorcraft with a cruise speed exceeding $220 \mathrm{kt}$. The compound architecture consists of a joinedwing lifting system supporting two pushing propellers providing thrust, yawing control and balancing the torque generated by the main rotor, (Figure 1). A mechanical transmission transfers power from the two turboshaft engines under the main rotor to the lateral propellers. Each upper wing (UW) structure, therefore, houses a drive shaft and relative transmission connections inside. An H-shape tail was selected to improve lateral manoeuvrability and reduce the susceptibility to rotor and wing wakes (Figure 1). New technologies were implemented to enhance the aircraft efficiency as advanced composite structural components, optimised transmission architecture and high voltage DC generation [1]. The 50\% increase in cruise speed compared to conventional helicopters enables the RACER to extend the accessible area within an hour flight for rescue and medical emergency missions as well as for passenger transport.

\section{RACER Joined-wing configuration}

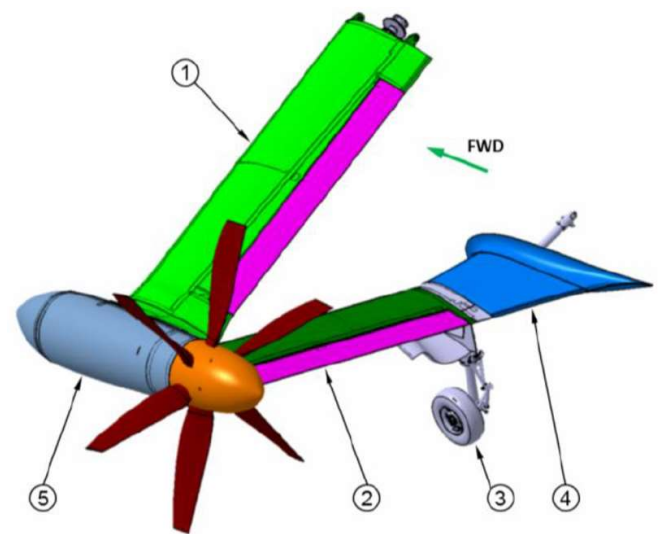

Figure 2: Racer joined-wing: wing configuration schematic (1 upper wing, 2 lower wing, 3 main landing gear, 4 stub wing, 5 lateral gear box nacelle) [1]

A joined-wing configuration was selected by $\mathrm{AH}$ to realise the lift compounding system of the RACER compound helicopter. The joined-wing is defined as a staggered bi-plane configuration with straight UW and lower wing (LW) structures at each side of the helicopter, being connected at their tip as shown in Figure 2. UW and LW structures feature opposed dihedral and sweep angles with a positive stagger arrangement at their roots, forming a triangular framework in both front and top views. The upper wing is connected 
to the upper fuselage whereas the lower one to the stub wing part of the lower fuselage. Lateral pushing propellers are located at the wing joint region, behind the trailing edges, offering improved characteristics in terms of passenger safety and crash-worthiness [1]. Joined-wings are generally characterised by higher aerodynamic efficiencies than traditional wing configurations due to the reduced induced drag, along with an increase structural efficiency owing to the global deformation behaviour of the double wing structure $[2,14]$. The joined-wing shows considerably larger stiffness on the vertical plane (flapping plane), but lower stiffness in the horizontal plane (feathering plane), in comparison with a traditional cantilever wing of equal total lift. This is consistent with the higher inertial and aerodynamic loads acting on the wing longitudinal plane of the compound helicopter and the stiffness requirement on the longitudinal plane imposed by the drive shaft and drive system deflection limits [1]. Torsional stiffness is enhanced by the wing connection and the staggered wing arrangement, which converts a portion of the global torsion into single wing bending. The joinedwing architecture reacts to bending loads on the longitudinal plane as a triangular beam system, with the wings mainly subjected to tensile and compressive loads. Additional superimposed local bending, shear and torsion stress characteristics are introduced by the aerodynamic lift distribution on the wing skin, flap actuations and propeller thrust.

The axial nature of the upper and lower wing loadings is an indication of structural efficiency and promotes weight reduction, as the entire wing section contributes to load carrying. Owing to the reduced portion of bending load carried, the suggested joined-wing configuration does not required structural continuity inside the fuselage as conventional cantilever wings do and the wing to fuselage attachment can be realised by simple hinges, which transfer transverse and longitudinal loads only. This minimises the space allocation of the wing structure in the upper fuselage deck where the engines and the main gearbox are housed [1]. Moreover, the joinedwing distributes the lifting and propulsion loads between the upper fuselage deck and the subfloor structure, reducing local structural stresses and mass of such fuselage components. The upper wing is essential for housing the propeller drive shaft and its sweep and anhedral angles are consequence of the respective positions of the main gearbox and the lateral propeller (Figure 2). The geometrical characteristics of the lower wing are mainly defined by the propeller location and the position of the stub wing in turn determined by the position of main landing gear, which is housed in the stub wing when retracted. Those constraints result in the peculiar staggered configuration with a lower wing positioned aft of the upper wing at their roots.
In addition, the $\mathrm{LW}$ provides a physical barrier between the passenger area and the rotating lateral propellers, acts as additional buoyancy body in case of ditching and as safety measure preventing the upper wing from breaking and obstructing the cabin door in case of crash [1]. From an aerodynamic perspective, no efficiency increase is achieved in this configuration due to the non-slender wing geometries and the airflow disruption caused by the main rotor and the lateral propellers. However, the wing staggering causes a favourable aerodynamic interaction between the wings and reduces the download of the downwash during hovering.

The joined-wing assembly consists of UW, LW and Nacelle structures. Each UW or LW structure includes a flap for trimming and stability purposes. The UW and LW structures interface with the fuselage separately via hinges, identified in Figure 3. The two wings are joined at their outboard tips through a connecting structure, known as a Cradle, which also supports the Nacelle. The Cradle is part of the UW structure and features two lugs to realise the connection with the LW. Spherical bearings and sliding bushes are utilised at various locations to mitigate translations and deformations of the wing structures relative to each other, and to the fuselage during flight operations. A schematic of the RACER wing architecture is shown in Figure 3. Connecting the wings using spherical bearings ensures an isostatic behaviour on the wing system vertical plane and the hinge position was optimised to maximise the stiffness of the joined structure. Loads along the helicopter longitudinal axis direction are transferred from the wings to the fuselage using dedicated X-trusses, allowing the hinges to carry only lateral and vertical shear. The gearbox located within the Nacelle, driving the propeller, is mechanically connected to the UW component of the wing to wing joint by an isostatic arrangement. Axial load is reacted by a dedicated X-truss between the gearbox and the Cradle within the UW structure.

Preliminary FE and CFD analyses confirmed the joined-wing as an efficient solution for a high-speed helicopter based on the compound formula with lateral propellers such as the RACER. However, the design and assembly of a statically indeterminate structure as a joined-wing presents some additional challenges compared to conventional, cantilever wings. The novelty of the aircraft and wing concepts prevents the use of standard assembly techniques and previous experience to build a structure compliant with the required tolerances. The weight optimised design of each wing structure also results in compliant and highly deformable components requiring supporting fixtures during handling and installation. In this scenario, innovative building solutions and analyses have to be implemented to develop an efficient and robust assembly process for the nonconventional, RACER joined-wing structure.

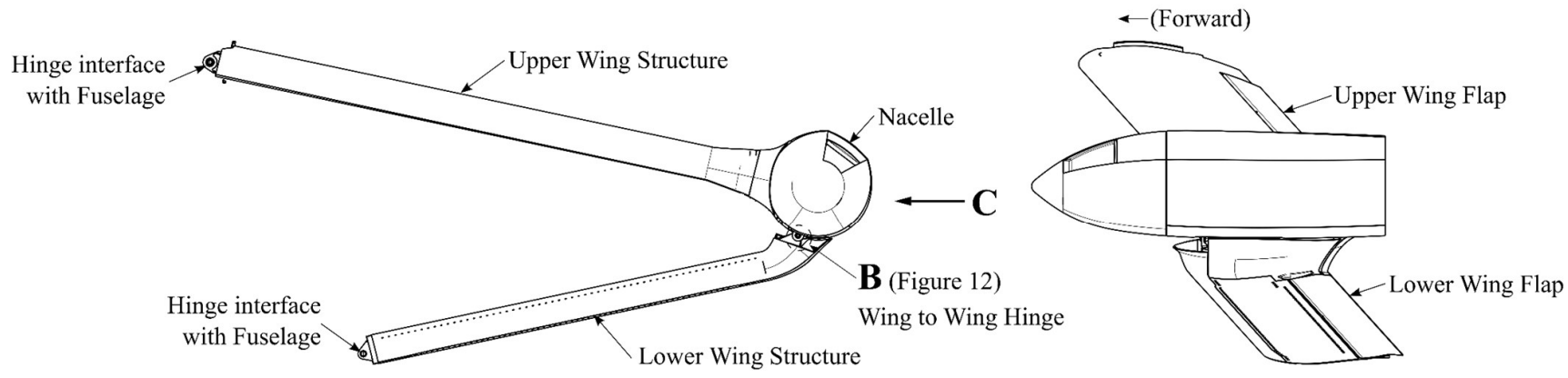

View looking rearwards on LHS Joined Wing Assembly in flight orientation

View on arrow $\mathbf{C}$ illustrating wing sweep

Fairings not shown where neccesary to identify hinge positions

Figure 3: RACER joined-wing assembly overview

Page 3 of 10 


\section{RACER joined-wing datums and interface}

Assembly interface requirements $[15,16]$ for the joined-wing configuration, shown in Figure 3, are defined relative to the individual wing structures rather than the joined-wing assembly as a whole. This is because the defined tolerances for the UW and LW structure geometries suitably bounds permutations of the joined-wing arrangement. The datum methodology of each wing structure is common to both the UW and LW. The physical features establishing datum A, B and C are the fuselage hinge bore, the aft face of the forward fuselage lug and the wing to wing hinge bore respectively, as depicted in Figure 4. The position of datum $\mathrm{C}$ directly controls the position of the lateral rotor, which is mounted to the outboard structure of the UW, within the Nacelle. The selected datum features support the design intent of the joined-wing architecture but add complications to the build process that are not present during the build of a typical fixed wing.
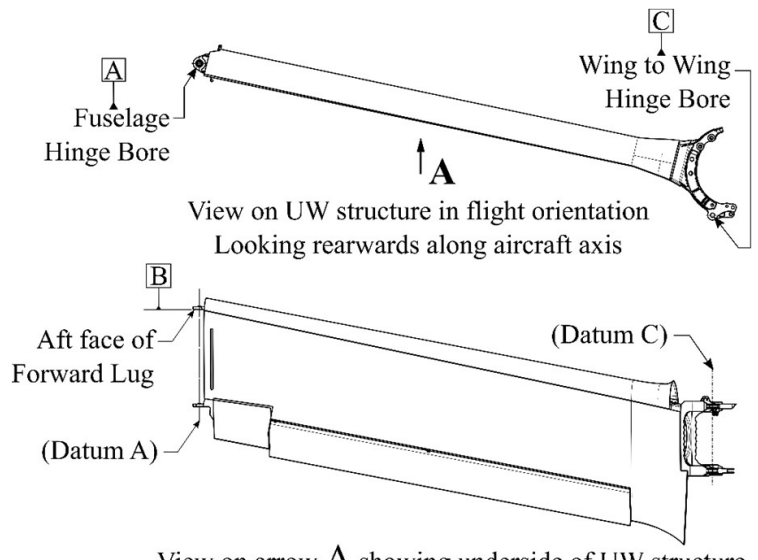

View on arrow A showing underside of UW structure

Figure 4: Wing structure datum architecture

A fixed, cantilevered wing structure will typically only feature an interface requirement for the inboard, root geometry interfacing with the fuselage. The primary complication with the assembly of the RACER wing design is that there is a requirement on the position of the inboard and outboard extents of each wing structure. The RACER interface requirements $[15,16]$ detail that the acceptable tolerance on the position of the datum $C$ bore, of each wing structure, is $\varnothing 0.2 \mathrm{~mm}$ relative to the Datum A and B of the wing structure in question. The $\varnothing 0.2 \mathrm{~mm}$ positional tolerance on the datum $\mathrm{C}$ bore impacts the design which must therefore wash-out tolerances, from the wing root and tip, towards the middle of the wingspan. This approach differs from that of a traditional wing design where tolerances can be run-out in a single direction, from the root towards the wing tip. The $\varnothing 0.2 \mathrm{~mm}$ positional tolerance also creates challenges within the assembly process. For example, a steel jig structure locating the upper wing would experience approximately $0.1 \mathrm{~mm}$ of thermal growth between features locating datums $\mathrm{A}$ and $\mathrm{C}$, due to a two degree temperature change. It should be noted that the right hand side (RHS) and left hand side (LHS) joined-wing structures are perfect mirror images of each other. Therefore, there are four independent wing structures to be built for the RACER demonstrator given that each joined-wing assembly features an UW and a LW structure.

The wing of a conventional, passenger carrying aircraft features a number of sub assemblies including slats, the wingbox, ailerons, spoilers and flaps; where Slats and Flaps are mounted to the forward and aft faces of the wingbox respectively. The wingbox itself is commonly assembled from a combination of Spars, Ribs, Stringers

Page 4 of 10 and Skins [17] arranged as shown in Figure 5a. This minimises weight whilst maximising stiffness. In comparison, the RACER wing structures do not require slats and the design has been optimised accordingly by GE Aviation Systems.

The independently built UW and LW structures of the RACER rotorcraft feature a common design philosophy based around the onepiece composite part that forms the upper aerodynamic surface and the Leading Edge (LE) 'D-nose', as identified in Figure 5b. Known as the Upper Cover, this part is the key component in the novel architecture of each wing structure. The co-moulded 'T-returns', to which the L shaped spars are fastened, contribute to the longitudinal stiffness performance of each structure whilst the sandwich structure of the panel increases stiffness such that riveted stringers are not required. Each RACER wing structure also features the parts listed below:

- Two L-shaped composite spars, Fore and Aft;

- Metallic Root Ribs featuring lugs to mount to the fuselage;

- Metallic components to facilitate the wing-to-wing hinge joint. Note; in the UW, this is the Cradle component described previously.

- Metallic Outboard Ribs in the LW only. Note; these are not required in the UW structure as the Cradle delivers the function.

- Two metallic Mid Ribs;

- Three metallic Trailing Edge (TE) Brackets to position the Wing Flaps;

- A composite Lower Cover, integrally stiffened and forming the lower aerodynamic surface.

\section{Assembly of the UW and LW Structures}

The largest components within each RACER wing structure are thin, composite parts that are relatively compliant in their free state. The remaining components are metallic, titanium or aluminium, items that are employed at locations requiring high precision, mechanical interfaces such as the wing to fuselage or wing to wing hinges, where bearings are to be pressed into bores.

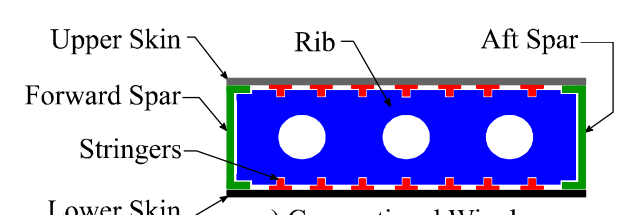

a) Conventional Wingbox

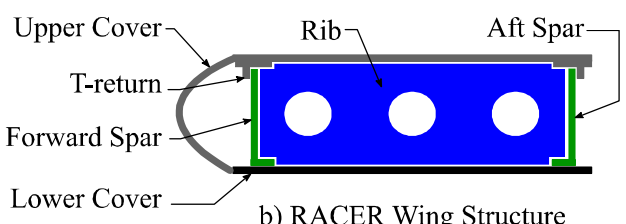

Lower Cover b) RACER Wing Structure

Figure 5: Diagrammatic comparison of a) conventional wingbox and b) the RACER wing structure

When designing an assembly process and necessary tooling for a demonstrator project, a determinate assembly methodology is preferable because it will reduce assembly cost, duration and tooling commissioning time [5]. Therefore, this approach was initially investigated for the build of the RACER wing structures. As noted previously, the determinate approach can increase part manufacturing cost if assembly processing is to be completely removed. Therefore, a compromised approach was proposed where parts would be 
accurately located to each other using the minimum number of precision-machined fastener holes; the remaining fastener holes would be produced during the assembly sequence. Unfortunately, the innovative design of the Upper and Lower Cover parts increased manufacturing cost such that including precision machined locating features, for determinate assembly, was deemed not possible. Therefore, the RACER wing structures are assembled using jigs to accurately set the position of all child items and hold the form of compliant composite items. The conceptual design of the jig structures was completed concurrently with the maturing of the build philosophy.

Whilst the design philosophy of the RACER UW and LW structures is based around the Upper Cover component, the part itself is not used to establish the datum structure of each wing, as noted in Figure 4. Therefore, the first part of each structure to be located in the jig is the Root Rib component as it establishes datums A and B (Figure 4) of the entire wing. The Upper Cover is then located in the jig using pick-ups along the leading edge and upper aerodynamic surfaces. These jig features hold the profile of the Upper Cover, preventing it from distorting during assembly or processing. The forward spar is then located and clamped to the forward 'T-return' item within the Upper Cover part. Until clamped or temporarily fastened into position, the spars are thin, highly compliant items. The outboard, wing to wing hinge components that establish datum $\mathrm{C}$ (Figure 4) are the next components to be installed in the jig before the aft spar is slotted between the aft T-return and the hinge components. This effectively closes the span-wise box structure and establishes the entire datum architecture for each wing. Whilst the outboard hinge items establish datum $\mathrm{C}$, it would not be possible to install the hinge items before the forward Spar and Upper Cover are in situ. Mid ribs are then located using pick-ups on the jig structure and TE Brackets are located using the flap sub-assembly, as discussed later. TE mouldings, closing gaps between the Upper and Lower Cover are then installed, located using edge of part interfaces. The Lower Covers are the last items to be located and fastened as required in each wing structure. An overview of the full wing structure assembly sequence is detailed in Figure 6 for reference.

Whilst the component datum systems have been defined to ensure datum to datum interfacing where possible, shim gaps are necessary in the design to mitigate manufacturing tolerances of child parts. To reduce the overall build duration, the shim application and curing stage will occur concurrently for all shim gaps. Therefore, the entire wing structure will be assembled and temporarily fastened, with gaps measured as items are assembled, before being stripped and cleaned. Release agent and shims will be applied where required, the entire structure will be re-assembled as previously detailed and the shim will be allowed to cure. The wing build facility at the University Of Nottingham is not thermally stable and therefore there is a risk of relative thermal displacements between the steel jig and the primarily composite wing. This will be mitigated by leaving the facility heating on constantly, with a thermostat, during the curing phase such that the temperature will remain constant within $\pm 1^{\circ} \mathrm{C}$. Once the shim has cured, the heating will be restored back to standard operating times. The pick-ups on the jig locating the outboard can then be 'unlocked' so that they still locate the hinge items but can translate in the plane of the jig, to mitigate jig thermal expansion. Whilst not a production standard solution, this does provide a cost effective solution for the RACER demonstrator, ensuring that thermal compensating features do not need to be included in the jig designs and meaning that a thermally stable assembly area is not required. The latter is costly to setup and maintain for a single demonstrator build.

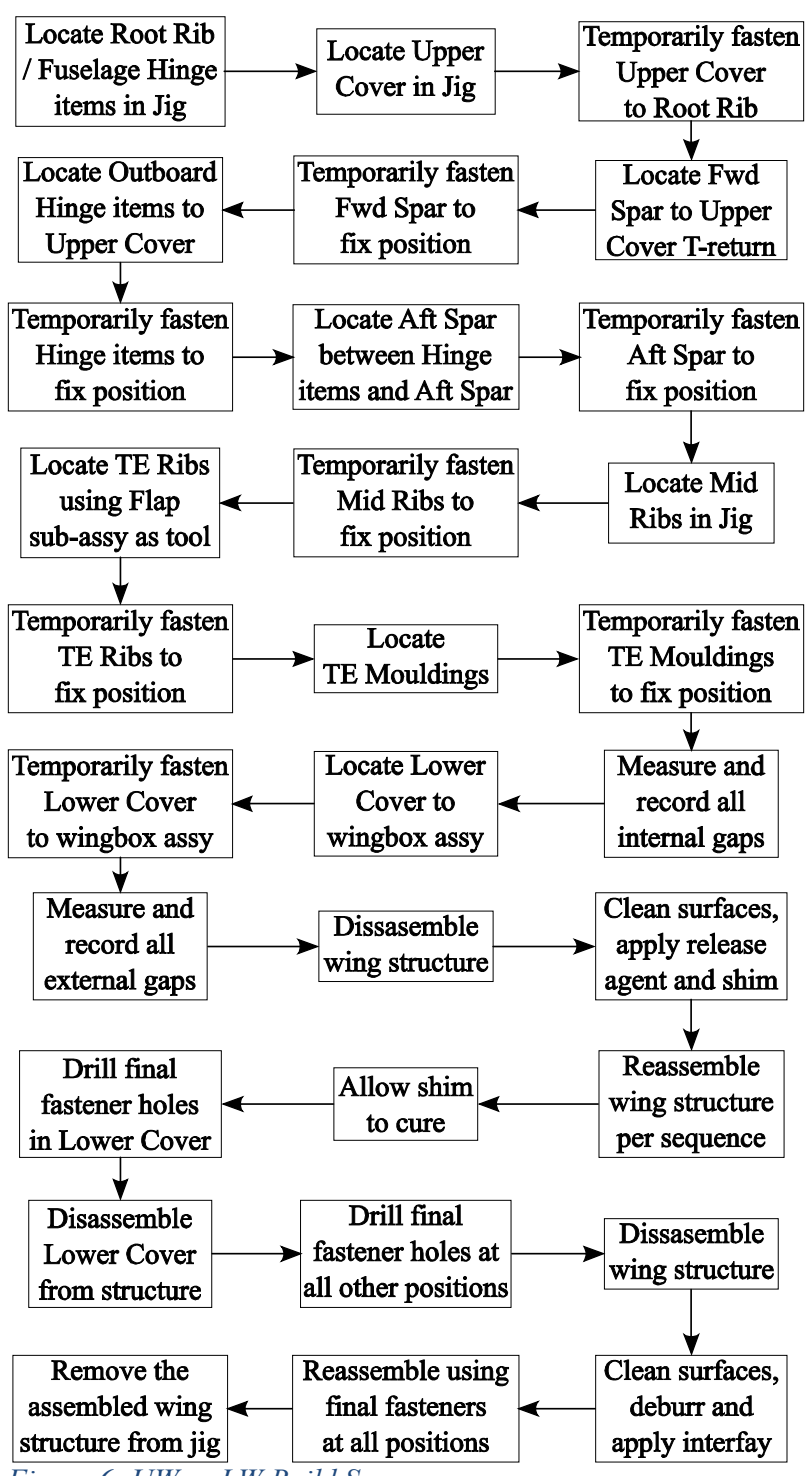

Figure 6: UW or LW Build Sequence

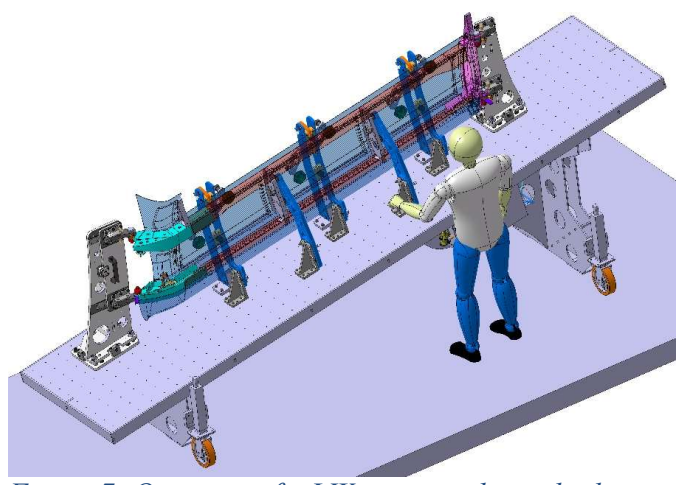

Figure 7: Overview of a LW structure being built in an Assembly Jig, courtesy of Electroimpact Ltd.

The required jig structures have been conceptually designed in parallel with the build philosophy. Detailed design and verification has been completed by Electroimpact Ltd. An image of the jig structure produced by Electroimpact Ltd. is depicted in Figure 7 showing a LW structure being assembled. The jig base shown 
appears oversized relative to the LW structure being assembled. This is because the $\mathrm{UW}$ is longer than the LW and the jig base is common to both the UW and LW structure jigs to minimise design effort and reduce cost for the demonstrator programme. The jig base plates feature a pattern of tapped holes across the entire supper surface. This allows the wing designers flexibility to adjust the wing geometry whilst low risk, high lead-time items of tooling, such as the jig bases, are being manufactured. This design feature mitigates the risk of late design changes whilst maximising concurrent engineering principles to reduce programme overall duration. This also makes the jigs reusable for projects of a similar nature or scale, or future iterations of the RACER demonstrator.

\section{Assembly of the Wing Structure Flaps}

Fixtures for assembling wing structures often feature a framework or structure that envelopes the product in one plane, to which the component pick-ups are fixed, to accurately locate child components [6]. Using a wingbox structure as an example, a surrounding fixture enables the concurrent assembly of leading and trailing edge items into the wingbox product whilst setting the wing to fuselage interface too. As noted previously, the RACER UW and LW assembly jigs will feature a frame around the LE, inboard and outboard extents of the wings. Each UW or LW structure features TE Brackets to locate the respective Flap sub-assembly. The interface requirements detail that the flap axis concentricity and overall flap position, relative to the wing datum structure, should be controlled to $\varnothing 0.3 \mathrm{~mm}$ and $\varnothing 0.5 \mathrm{~mm}$ respectively $[15,16]$.

A jig structure fully surrounding the wing product was considered to locate the TE Brackets, per typical assembly philosophies, as shown in Figure 8. This style of jig would facilitate the following flap assembly sequence:

1) Locate TE Brackets to the wingbox using pickups from a surrounding frame style main assembly jig;

2) Shim, drill and fasten the TE Brackets to the wingbox as required

3) Locate Flap Ribs to TE Brackets using tooling or fasteners;

4) Slide the Flap Panel onto Flap Ribs, along vector of Flap Axis;

5) Shim, drill and fasten the Flap Panel to the Flap Ribs.

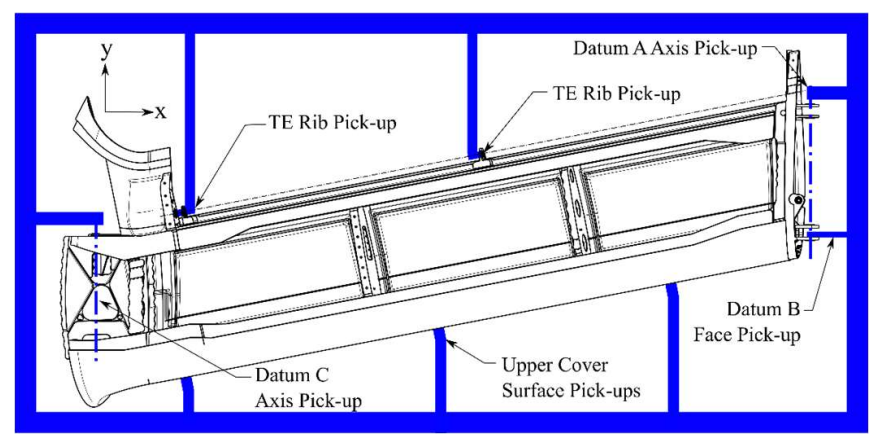

Figure 8: Surrounding Jig Structure Concept to assemble LW Flap items

A detailed review of this typical assembly sequence, with respect to the RACER Flap geometry, highlighted a few constraints. The RACER Flap Panels are one-piece composite mouldings, which enclose the flanges of the Flap Ribs in a C-shaped profile. The Flap Ribs also feature hard stops to prevent rotation of the assembled flap exceeding $11^{\circ}$ in either direction, about the flap axis. These two design features are likely to prevent the completion of task 4 , in the above sequence without the Flap Panel clashing with another

Page 6 of 10 component. Additionally, given the small scale of the RACER Flap items, there will be very limited access to complete task 5 in the above sequence once the Flap Panel is located to the wingbox.

A further constraint with the identified sequence relates to the style of the jig structure required and the necessity to build the wing structures in an environment with an unstable temperature. The surrounding style jig structure, conceptually depicted in Figure 8, is subject to thermal expansion due to temperature fluctuations in both the $\mathrm{X}$ and $\mathrm{Y}$ directions. As noted previously, the majority of components within each RACER wing assembly are composite with negligible thermal expansion. By employing a steel, surrounding frame style jig subject to a temperature fluctuation of $\pm 2^{\circ} \mathrm{C}$, the position of a Flap item could vary by up to $0.1 \mathrm{~mm}$ and $0.04 \mathrm{~mm}$ in the $\mathrm{X}$ and $\mathrm{Y}$ planes respectively, relative to the datum system of each wing structure. In reality, it is expected that the assembly facility temperature could fluctuate by up to $10^{\circ} \mathrm{C}$ throughout a 24 hour period. This would have a significant impact on the concentricity of flap axis items were the temperature to fluctuate whilst the shim was curing.A final constraint of a surrounding frame style jig structure is the cost. The four wing structures within the RACER aircraft will each be assembled in a bespoke jig and each wing structure includes a Flap sub-assembly. Adding features to the four wing structure assembly jigs to control the TE Bracket position would increase the overall cost of the jigs dramatically.

Therefore, an optimised assembly philosophy was developed, listed below, to overcome the constraints detailed.

1) Assemble the Flap Brackets and the Flap Panel into a subassembly using a separate, reconfigurable jig;

2) Shim, cure, drill and final fasten the Flap Brackets to the Flap Panel in the sub-assembly jig;

3) Using the assembled Flap sub-assembly as the tooling, locate the TE Brackets to the wingbox assembly;

4) Shim, cure, drill and final fasten the TE Brackets to the wingbox.

This optimised build philosophy is realised using a reconfigurable assembly jig, capable of assembling all four flaps within the RACER rotorcraft. The jig, depicted in Figure 9, is sufficiently compact to allow it to be used in one of the University of |Nottingham's thermally stable research laboratories, negating any effect of thermal coefficient mismatches between the steel jig structure and the composite Flap Panel. The Flap sub-assemblies produced are stiff, light and thermally matched to the primary wingbox structures and therefore ideal to use as tooling to set the location of the wingbox TE Ribs. This strategy removes issues regarding drill and fastener access to the Flap Ribs, whilst fastening to the Flap Panel. The optimised philosophy is possible given that the Flap sub-assemblies can be located to components within the primary wing structures via accurately machined interfaces. This ensures that the $\varnothing 0.5 \mathrm{~mm}$ tolerance on the flap axis position, relative to the wing datum system, will be achieved.

The $\varnothing 0.3 \mathrm{~mm}$ concentricity tolerance on the flap axis item is achieved through effective detailing of the jig items, to minimise tolerance impacts, and the precision machining of the tooling items, such as brackets and pins, to micron level accuracy. The flap sub-assembly jig can then be certified through the presentation of tolerance stackups and jig parts manufacturing inspection data, which reduces setup time by removing the need to track the flap tooling into position using intelligent metrology systems. As the RACER is a demonstrator project, cost and programme duration are to be reduced wherever possible and the optimised flap build philosophy supports 
this intention. The manufacture of the jig items will be significantly cheaper than the cost required add features to the four wing assembly jig structures. Secondly, the use of the reconfigurable flap jig allows the flap sub-assemblies to be assembled concurrently with the primary wing structures, minimizing the overall build duration.

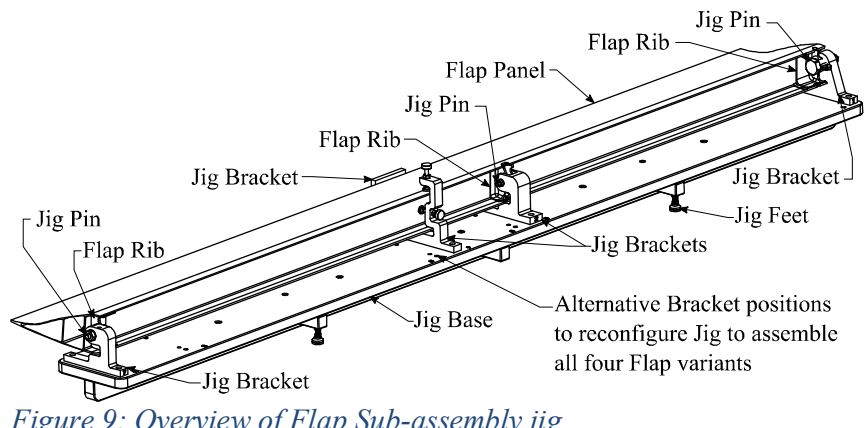

\section{Joining of the UW and LW structures}

Aerospace hinged joints are typically assembled with a part to part methodology or using adjustable connecting rods so that no drilling of reaming of the hinge components is required during assembly. The tolerance on the position of the outboard hinge lines is $\varnothing 0.2 \mathrm{~mm}$, relative to the datum system of each wing structure. Whilst a tight tolerance for the assembly of a wing structure, this is not sufficient to allow a part-to-part assembly philosophy at the hinge given the tolerances and clearances required of the pins, bushes and bearings in a hinged joint. In addition, the distance between the inboard and outboard hinge lines of each wing structure cannot be adjusted once assembled. Therefore, it is necessary to drill and ream hinged joints within the RACER joined-wing configuration, during the assembly sequence, to produce concentric bores through all interfacing components with the required tolerances for the connecting pins. The wing to fuselage joints at both the upper and lower positions feature spherical bearings in one side of the interface whereas the wing-towing hinge joint features a combination of headed and sliding bushes. Therefore, the wing-to-wing joint is to be processed during assembly given the difficulty associated with reaming a spherical bearing.

\section{Assembly sequence and matched tooling}

To ensure that the final wing assembly will fit to fuselage, matched tooling was suggested by AH. It is typically employed in applications where it is challenging to assemble either side of an interface independently without reference of the interfacing geometry; or where an assembly process for either interfacing structure may not deliver the tolerances necessary to achieve complete interchangeability. The matched tooling is required in the RACER wing to fuselage application because some tasks within the joinedwing assembly procedure, such as locating, shimming and fastening the nacelle fairings, can only be completed with the wings located as assembled to the fuselage. Whilst these processes could be completed during final assembly of the wings to the fuselage, completing the processes prior to meeting the completed fuselage will reduce overall assembly duration. In this application, the matched tool will replicate the wing to fuselage interface with the hinge lines at the wing to wing joint produced by a common boring operation to ensure concentricity. The tool can then be split so that the wing assembly process can be completed using the fuselage replica tool and vice versa. For reference, Figure 10 illustrates an overview of the joined-wing assembly sequence.

Page 7 of 10

Page 7 of 10
The opportunity to employ a modular matched tool was presented which would allow the fuselage replica tool to be split, creating separate fuselage hinge line modules for the UW and LW. These modules could be utilised during the independent assembly of the UW and LW structures. It was expected that the separate hinge line modules could be incorporated into reconfigurable jig structures [6] that were designed and validated in parallel with the activities presented in this paper. The obvious benefit to this opportunity is that the individual wing structures would be assembled to an exact replica of the fuselage hinge hardware. However, this may not be an issue if both the matched tooling and the individual wing structures can be produced to meet the interface requirements. The obvious disadvantage of a modular system is that the sum of tolerances affecting the position of one fuselage hinge line, relative to the other, will be greater than for a non-modular tool but the affect is not easily quantified. A thorough discussion on the two matched tooling concepts and the framework developed to select the most appropriate one can be found in [13].

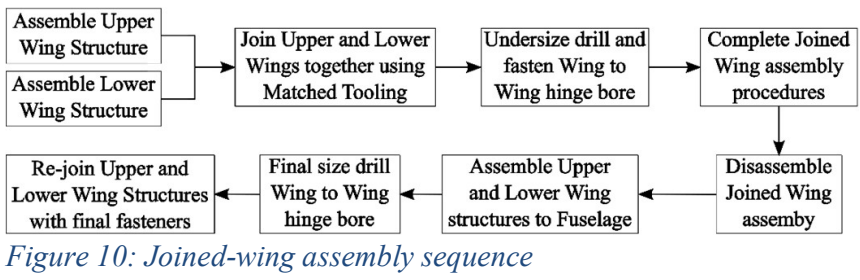

\section{Assembly Key Characteristic}

Figure 10 details that the wing to wing hinge joint will be bored undersize whilst the wings are mounted to the matched tooling, and then bored full size when finally mounted to the fuselage. Drilling a hinge bore during assembly ensures the bore is concentric through all interfacing components, removing the effect of assembly tolerances. The first, undersize boring will mitigate tolerances arising from the matched tooling but also from the assembly of the individual wing structures. The second, final size bore will mitigate any minor deviations that may arise between the replica matched tooling, as shown in Figure 11, and the real fuselage or joined-wing structures. In the RACER wing-to-wing hinge application, the interfacing components will experience relative translations and rotations during assembly and flight conditions. Headed bushes are used at the hinge joint, as shown in Figure 12, to minimise wear of the parent materials during relative displacements. The sliding bushes react the bolt tightening load axially, preventing transfer of the load to the female lug component, which could close the lug opening and limit relative translation of the male and female lugs. The headed bushes will be pressed into the parent components prior to assembling and joining the wing structures. Therefore, material will be removed from the inner diameter (ID) of all eight headed bushes during the two boring processes noted in Figure 10.

Drilling the headed bushes during the joined-wing assembly process is preferable to drilling the parent components and inserting fixed size bushes. This is because it mitigates risks regarding damaging expensive parts such as the hinge components whilst completing manual drilling tasks. The headed bushes within the female lug, shown in Figure 12, feature a larger diameter bore to locate the sliding bushes which will not be bored during assembly of the wings. Boring of the headed bushes will be completed with a stepped drill bit, to produce concentric bores in bushes within both lugs simultaneously. 

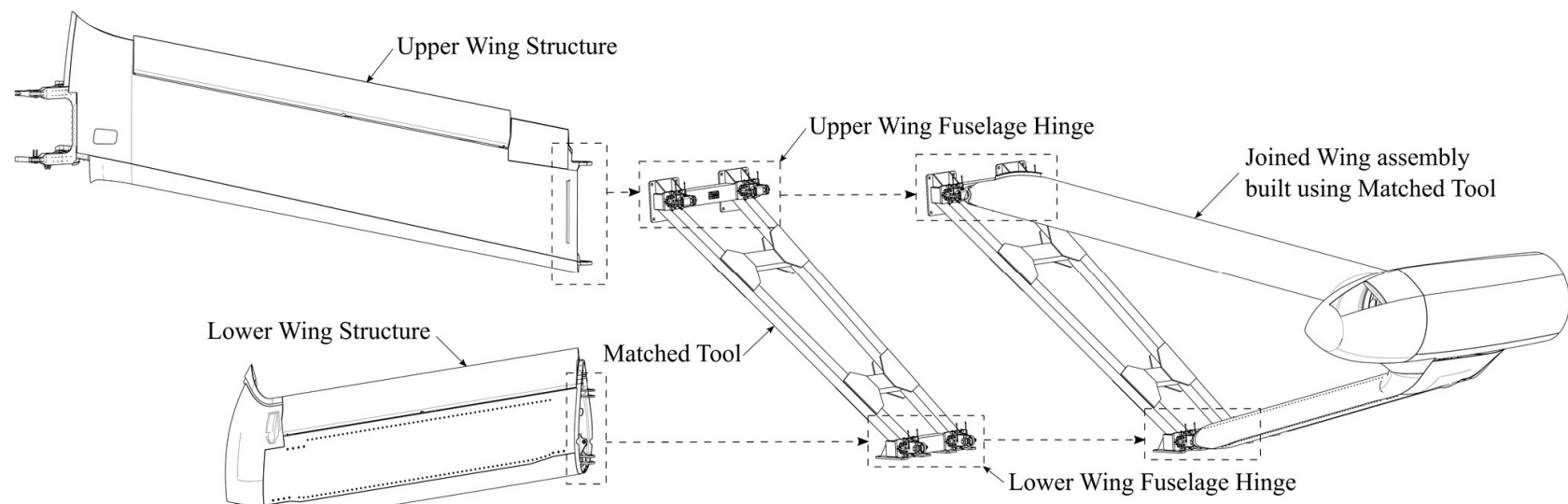

Figure 11: Fuselage Replica Matched Tooling Concept

The bores in one of the headed bushes located in the female lug, and the two bushes located in the male lug will be produced in a single drilling procedure, normal to the surface of the female lug parent component as detailed in Figure 12. The bore in the remaining headed bush in the female lug will be produced by drilling back in the opposite direction through the female lug, using the new bore in the male lug to locate the drill bit. Boring bushes in the interface during assembly requires the ID of the pre-bored bushes to be sufficiently undersized so that a clean hole can be drilled through regardless of lug misalignment. If the ID of a bush before boring is too large, the bored hole may not remove material through the entire length of the bush. This can lead to increased bush wear and reduced bush life as radial loads will not be distributed as designed. However, a smaller bush ID means that more material will need to be removed from the bush, increasing assembly duration and the transfer of thermal energy to the structure; which is typically avoided where possible. Therefore, the ID of a pre-bored bush should be optimised by $3 \mathrm{D}$ tolerance analyses to minimise the material to be removed whilst ensuring a hole can be bored through the entire bush thickness, for all lug misalignment conditions.

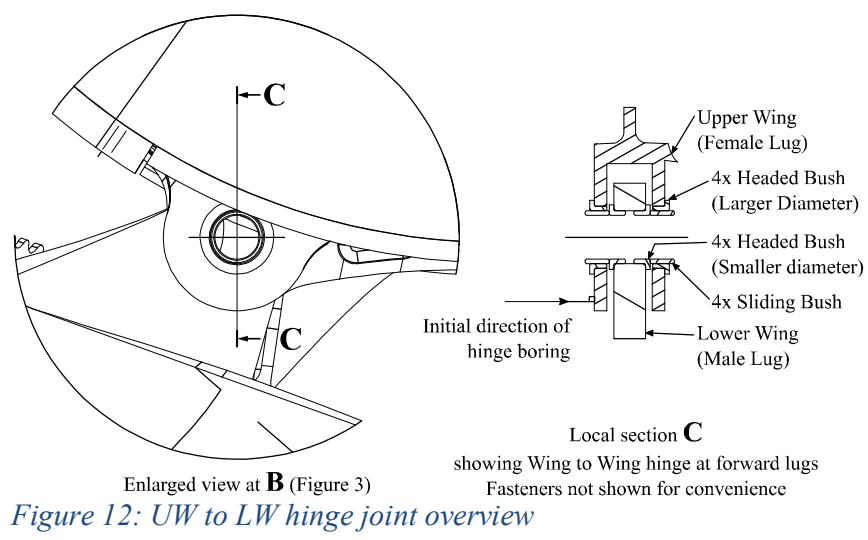

\section{D Tolerance analysis}

Quantifying the impact on the required pre-bored bush ID is a complex, 3D problem that is not easily quantified using traditional, two-dimension tolerance stack-ups. Variation propagation analysis should be applied using 3D tolerance techniques. Generally, a mechanical assembly can be described mathematically by assuming reference frames at the various parts, or subassemblies, as well as at the assembly features on the parts [4]. Homogeneous Transformation
Matrices (HTM) are used to describe frames. An assembly is modeled as a chain of these frames. Chaining all the different coordinate systems by multiplying appropriate transforms ${ }^{n} T_{j}^{i}$, obtains an assembly model [4]. The homogeneous transformation matrix, ${ }^{n} T_{j}^{i}$ from the frame $i$ to the frame $j$ is given by

$$
{ }^{n} T_{j}^{i}=\left[\begin{array}{cc}
R_{j}^{i} & p_{j}^{i} \\
0^{T} & 1
\end{array}\right]
$$

where the leading superscript $n$ indicates nominal form (no variation) and $R_{j}^{i}$ is the $3 \times 3$ rotation matrix and $p_{j}^{i}$ the $3 \times 1$ translation vector. In the varied form, the transformation matrix $T_{j}^{i}$ is multiplied with the appropriate matrix $D T_{j}$ indicating variation and a new transform is calculated by

$$
{ }^{v} T_{j}^{i}={ }^{n} T_{j}^{i} \cdot D T_{j}
$$

where $D T_{j}$ is given by

$$
D T_{j}=I_{4 \times 4}+\left[\begin{array}{cccc}
0 & \delta \theta_{z} & \delta \theta_{y} & d x \\
-\delta \theta_{z} & 0 & -\delta \theta_{x} & d y \\
-\delta \theta_{y} & \delta \theta_{x} & 0 & d z \\
0 & 0 & 0 & 0
\end{array}\right]
$$

where $\delta \theta_{x}, \delta \theta_{y}$ and $\delta \theta_{z}$ are small rotations and $d x, d y$ and $d z$ are small translations with respect to frame $j$, representing variation from the nominal form arising from manufacturing or assembly tolerances. The required pre-bored bush ID is estimated by tailoring the homogeneous transformation matrix method to the case study and implementing the following tasks:

- $\quad$ establishing an appropriate measure to quantify the pre-bored bush ID;

- $\quad$ assigning appropriate frames to the various parts and interfaces of the wing model;

- $\quad$ extracting homogeneous transforms from the wing CAD model;

- $\quad$ determining the assembly models;

- $\quad$ representing GD\&T tolerances with appropriate matrix format;

- considering the kinematic joints in the wing to fuselage interfaces that wash out part of the variation at the wing-towing interface;

- $\quad$ calculating the worst case scenario, i.e. the optimised bush ID.

Page 8 of 10 
Further details on the methodology and its application to the RACER joined-wing can be found in [13].

\section{Joined-Wing Assembly Jig}

Whilst the positional accuracy of the joined-wings will be ensured using the matched tooling, a jig structure is required to support the mass of the matched tooling and the wings during the joining and final assembly processes. The jig structure is not required to deliver any positional requirements and can therefore be produced efficiently using reconfigurable tooling, such as BoxJoint [6] supplied by Prodtex Ltd. The reconfigurable nature of the BoxJoint tooling system allows a structure to be produced quickly with minimal capital investment [6], which is ideal for the RACER demonstrator project. The structure required to facilitate the assembly of the joined-wing structure has been designed and detailed using hand calculations to underwrite the integrity. As shown in Figure 13, the jig structure is symmetrical, about a central plane, allowing the assembly of multiple joined-wings to be completed concurrently when desired.

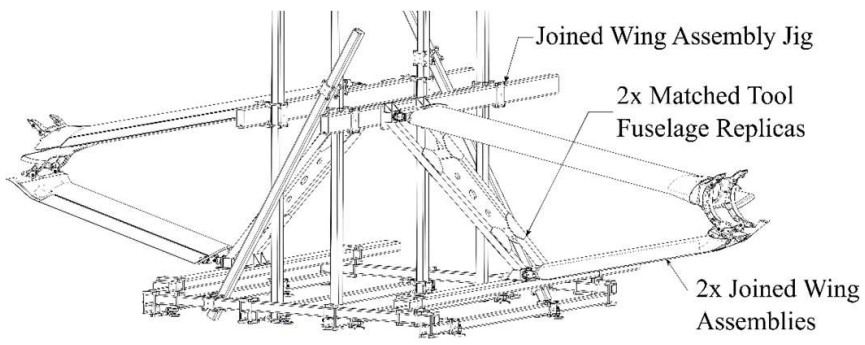

Figure 13: Joined-wing assembly jig overview

\section{Results and Discussion}

An optimised assembly philosophy has been developed to facilitate the build of the joined-wing structure for the RACER demonstrator. This has been achieved through detailed analysis of the design geometry, a thorough understanding of the assembly tolerances and child part datum systems, detailed design of bespoke tooling and the completion of 3D tolerance analyses using homogenous transformation matrices. The resultant build philosophy is summarised in Figure 14.

This work proves that constraints on the assembly process due to the complex joined-wing configuration can be overcome with cost effective solutions that minimise overall build duration. The work has produced robust, reconfigurable tooling designs that will be employed to support the single RACER demonstrator but, with some duplication, could also be utilised to support a production standard build rate.

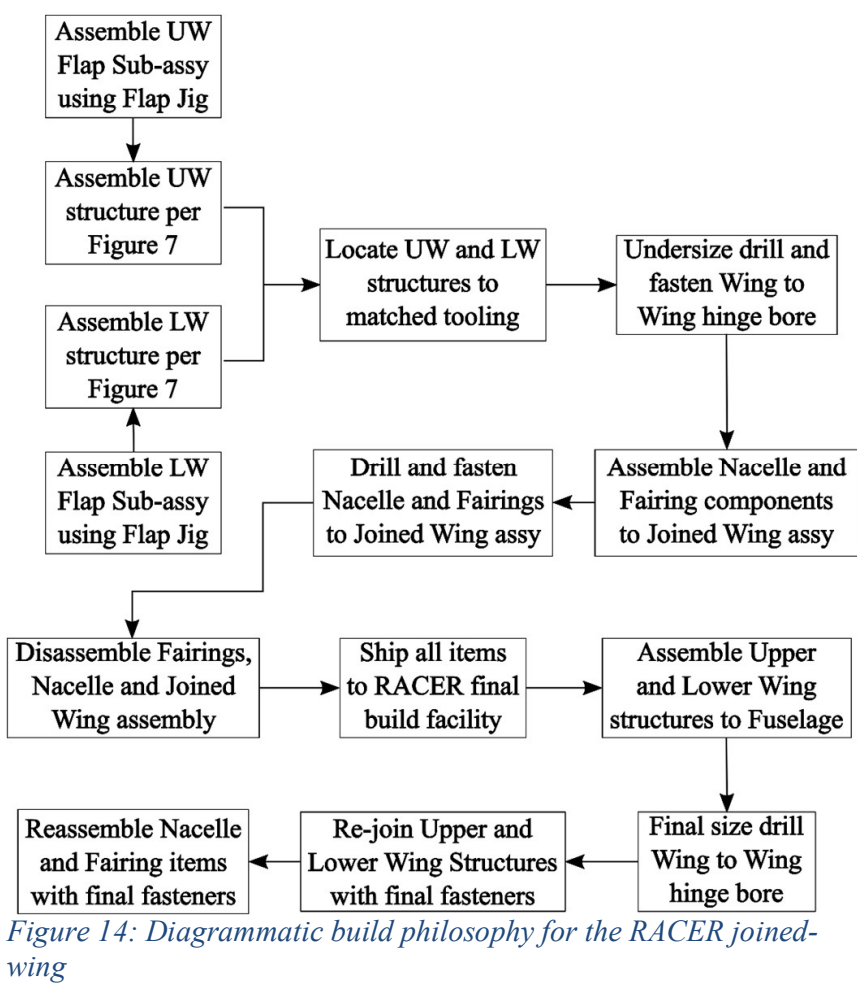

\section{Conclusions}

An industrial engineering problem with a tangible outcome was addressed by this work. Identifying the optimum strategy to assemble the RACER wing structure is of high importance and the solution detailed in this work overcomes the challenges associated with producing a joined-wing from numerous, composite, compliant components.

To develop the build philosophy, the engineering design was thoroughly reviewed, whilst still relatively immature, to capture the design intent and ensure that appropriate methods and tooling could be produced. The datum systems of components within the wing structures were identified to best support the assembly philosophy. An optimised assembly sequence was defined to reduce the overall duration; preventing component clashes during installation and ensuring ease of access for the insertion of fasteners. An innovative method of locating wingbox TE Ribs using interfacing Flap components was identified and a Flap sub-assembly jig has been designed and detailed to deliver the positional tolerances desired.

The challenge of successfully joining the two wing structures at their common hinge line has been overcome by utilizing matched tooling and boring the hinge line during the assembly process. The optimum inner diameter of the pre-bored bushes has been defined through homogenous transform methods. Overall assembly process cost has been minimised by using reconfigurable Flap tooling and through the use of the Boxjoint system to produce the joined-wing assembly jig. 


\section{References}

1. Blacha, M., Fink, A., Eglin, P., Cabrit, P., "Clean Sky 2: Exploring New Rotorcraft High Speed Configurations," presented at $43^{\text {rd }}$ European Rotorcraft Forum 2017, Italy, September 12-15, 2017.

2. Wolkovitch, J., "The Joined Wing-An Overview," Journal of Aircraft 23 (3):161-178, 1986, doi: 10.2514/3.45285.

3. Curran, R., et al. "Modelling of aircraft manufacturing cost at the concept stage." The International Journal of Advanced Manufacturing Technology 31.3-4 (2006): 407-420.

4. Naing, S. et al. "Design for Tooling to Enable Jigless AssemblyAn Integrated Methodology for Jigless Assembly." SAE transactions (2000): 299-311.

5. Irving, L., Ratchev, S., Popov, A., and Rafla, M., "Implementing Determinate Assembly for the Leading Edge Sub-Assembly of Aircraft Wing Manufacture," SAE Int. J. Aerosp. 7(2):246-254, 2014

6. Millar, A. \& Kihlman, H., Reconfigurable flexible tooling for aerospace wing assembly. No. 2009-01-3243. SAE Technical Paper, 2009

7. Whitney, D., "Mechanical Assemblies: their design, manufacture and role in product development," (Oxford University Press, 2004), ISBN 0-19-515782-6.

8. Islam, M., "Functional dimensioning and tolerancing software for concurrent engineering applications," Computers in Industry, 54: 169-190, 2004, doi: 10.1016/j.compind.2003.09.006

9. Laperrière, L., EIMaraghy, H., "Tolerance analysis and synthesis using Jacobian transforms,", CIRP Annals, 49(1): 359362, 2000, doi:10.1016/S0007-8506(07)62964-3

10. Marziale, M., Polini, W., "A review of two models for tolerance analysis of an assembly: vector loop and matrix," International Journal of Advanced Manufacturing, 43 (11-12): 1106-23, 2009, doi: 10.1007/s00170-008-1790-0

11. Ameta, G., Samper, S., "Comparison of spatial math models for tolerance analysis: Tolerance-Maps, Deviation Domain, and TTRS," Journal of Computing and Information Science in Engineering, 11: , 2011, doi: 10.1115/1.3593413

12. Shen, S., Ameta, G., Shah, J., Davidson, J., "A Comparative Study of Tolerance Analysis Methods," Journal of Computing and Information Science in Engineering, 5: 247-256, 2005, doi: 10.1115/1.1979509.

13. Bacharoudis, K., Bainbridge, D., Turner, A., Popov, A., et al "3D Tolerance Analysis of a joined wing concept for a compound helicopter to aid tooling design selection," Submitted to Journal of Manufacturing Systems, 2019

14. Cavallaro, R., Demasi, L., "Challenges, ideas and innovation of joined-wing configurations: a concept from the pas, an opportunity for the future," Prog Aerosp Sci 87:1-93, 2016.

15. GE aviation, “ASTRAL WING UPPER ICY," RT570R7000, 2018

16. GE aviation, “ASTRAL WING LOWER ICY,” RT570R7001, 2018

17. Chun-Yung Niu, M., Airframe Structural Design, Chapter 8 Wing Box Structure, Conmilit Press Ltd, 1988

18. AMF Zero-point Systems Product Catalogue, www.amf.de/files/amfassets/news/downloads/catalogues/Catalogue-AMF-Zero-PointSystems.pdf, accessed April 2019

\section{Contact Information}

Corresponding Author: David Bainbridge

David.Bainbridge@nottingham.ac.uk

\section{Acknowledgements}

This project has received funding from the Clean Sky 2 Joint Undertaking (JU) under grant agreement number CSJU-CS2-GAMAIR-2014-2015. The JU receives support from the European Union's Horizon 2020 research and innovation programme and the Clean Sky $2 \mathrm{JU}$ members other than the Union.

GE are acknowledged for producing the detailed design of the joinedwing assembly, including all child parts, and the manufacture of all items.

Electroimpact Ltd. are acknowledged for their support of the build philosophy and the detailed design and manufacture of the four wing structure assembly jigs.

Zi Wang, University of Nottingham PhD student, is acknowledged for the detailed design and verification analysis of the Joined-Wing Assembly Jig (Figure 13). 